Appl. Set-Valued Anal. Optim. 3 (2021), No. 1, pp. 95-107

Available online at http://asvao.biemdas.com

https://doi.org/10.23952/asvao.3.2021.1.08

\title{
THE ITERATIVE ALGORITHM WITH INERTIAL AND ERROR TERMS FOR FIXED POINTS OF STRICTLY PSEUDOCONTRACTIVE MAPPINGS AND ZEROS OF INVERSE STRONGLY MONOTONE OPERATORS
}

\author{
D.F. AGBEBAKU ${ }^{1}$, P.U. NWOKORO ${ }^{1}$, M.O. OSILIKE ${ }^{1, *}$, E.E. CHIMA ${ }^{2}$, A.C. ONAH ${ }^{1}$ \\ ${ }^{1}$ Department of Mathematics, University of Nigeria, Nsukka, Nigeria \\ ${ }^{2}$ Department of Mathematics, Bingham University, Karu, Nigeria
}

\begin{abstract}
We study a Halpern-type algorithm with both inertial and error terms for the approximation of fixed points of strictly pseudocontractive mappings and zeros of inverse strongly monotone operators in real Hilbert spaces. Our algorithm is illustrated via numerical examples in both finite and infinite dimensional real Hilbert spaces. Our results extend recent results of [Y. Shehu, O.S. Iyiola, F.U. Ogbuisi, Iterative method with inertial terms for nonexpansive mappings: applications to compressed sensing, Numer. Algor. 83 (2020), 1321-1347] from the class of nonexpansive mappings to the much more general class of strictly pseudocontractive mappings.
\end{abstract}

Keywords. Strictly pseudocontractive mappings; Nonexpansive mappings; Halpern-type algorithm; Inertial terms; Strong convergence.

\section{INTRODUCTION}

Let $H$ be a real Hilbert space with inner product $\langle.,$.$\rangle and induced norm \|$.$\| . Let C$ be a nonempty closed convex subset of $\mathrm{H}$.

Definition 1.1. A mapping $T: C \rightarrow C$ is said to be L-Lipschitzian if there exists $L \geq 0$ such that $\|T x-T y\| \leq L\|x-y\|, \forall x, y \in C . T$ is said to be a contractive if $L \in[0,1)$, and $T$ is said to be nonexpansive if $L=1 . T$ is said to be demiclosed at $p$ if whenever $\left\{x_{n}\right\}_{n=1}^{\infty}$ is a sequence in $C$ which converges weakly to $x^{*} \in C$ and $\left\{T x_{n}\right\}_{n=1}^{\infty}$ converges strongly to $p$, then $T x^{*}=p$.

Let $P_{C}: H \rightarrow C$ denote the metric projection (the proximity map) which assigns each point $x \in H$ to the unique nearest point in $C$, denoted by $P_{C}(x)$. It is well known that $z=P_{C}(x)$ if and only if $\langle x-z, z-y\rangle \geq 0, \forall y \in C$, and that $P_{C}$ is nonexpansive.

Definition 1.2. A mapping $T: C \rightarrow C$ is said to be $k$-strictly pseudocontractive [1] if there exists $k \in[0,1)$ such that $\|T x-T y\|^{2} \leq\|x-y\|^{2}+k\|(x-T x)-(y-T y)\|^{2}, \forall x, y \in C$.

The class of $k$-strictly pseudocontractive mappings is more general than the class of nonexpansive mappings.

${ }^{*}$ Corresponding author.

E-mail address: dennis.agbebaku@unn.edu.ng (D.F. Agbebaku), peter.nwokoro@unn.edu.ng (P.U. Nwokoro), micah.osilike@unn.edu.ng (M.O. Osilike), epukeezinneuk@yahoo.com (E.E. Chima), onah87@gmail.com (A.C. Onah).

Received May 31, 2020; Accepted July 30, 2020. 
Definition 1.3. $T$ is demicontractive in the terminology of Hicks and Kubicek [2] or (satisfies condition A) in the terminology of Maruster [3] if $F(T)=\{x \in C: T x=x\} \neq \emptyset$ and there exists $k \in[0,1)$ such that

$$
\|T x-p\|^{2} \leq\|x-p\|^{2}+k\|x-T x\|^{2}, \quad \forall x \in C ; p \in F(T) .
$$

In the iterative approximation of fixed points of nonexpansive maps and strictly pseudocontractive mappings, the iterative schemes of Mann [4]:

$$
x_{n+1}=\left(1-\alpha_{n}\right) x_{n}+\alpha_{n} T x_{n}, n \geq 1 ;
$$

and Ishikawa [5]:

$$
x_{n+1}=\left(1-\alpha_{n}\right) x_{n}+\alpha_{n} T\left[\left(1-\beta_{n}\right) x_{n}+\beta_{n} T x_{n}\right], n \geq 1,
$$

where $\left\{\alpha_{n}\right\}_{n=1}^{\infty}$ and $\left\{\beta_{n}\right\}_{n=1}^{\infty}$ are suitable sequences in $[0,1]$, play an important role. However, the two iteration schemes yield the weak convergence only, and require the "compactness" assumption either on the operators or the domain of the operators or even both to yield the strong convergence. Often, strong conditions are needed to impose on the fixed-point set, $F(T)$, to obtain the strong convergence by using the Mann or the Ishikawa iteration process (see, for example, [6, 7]). For example, in [6], Qihou required that $F(T)$ is finite, where $T$ is a continuous pseudocontractive-type self-mapping of a nonempty convex compact of a Hilbert space, and, in [7], Zegeye, Shahzad and Alghamdi required that the interior of $F(T)$ is nonempty, where $T$ is a Lipschitz pseudocontractive self-mapping of a nonempty closed convex subset of a Hilbert space. Recently, many new schemes have been studied to achieve the strong convergence with mild assumptions on the operators, their domains, their fixed point sets and other necessary components (see, for example, $[8,9,10,11,12,13,14]$ ).

In [15], Shehu, Iyiola and Ogbuisi introduced and studied a Halpern-type algorithm with both inertial and error terms for approximating fixed points of nonexpansive mappings in real Hilbert spaces. They proved the following main convergence theorem.

Theorem 1.1. ([15, Theorem 4.2]) Let $H$ be a real Hilbert space and let $T: H \rightarrow H$ be a nonexpansive mapping with a nonempty fixed point set $F(T)$. Let $\left\{x_{n}\right\}$ be the sequence generated from arbitrary $x_{0}, x_{1} \in H$ by

$$
\left\{\begin{array}{l}
y_{n}=x_{n}+\theta_{n}\left(x_{n}-x_{n-1}\right), \\
x_{n+1}=\alpha_{n} x_{0}+\beta_{n} y_{n}+\gamma_{n} T y_{n}+e_{n}, n \geq 1,
\end{array}\right.
$$

where $\left\{\alpha_{n}\right\},\left\{\beta_{n}\right\},\left\{\gamma_{n}\right\}$ are sequences in $(0,1) ;\left\{\varepsilon_{n}\right\}$ is a positive sequence and $\left\{e_{n}\right\} \subseteq H$ is a sequence of errors, and these sequences satisfy the conditions:

(i) $\lim _{n \rightarrow \infty} \alpha_{n}=0, \sum_{n=1}^{\infty} \alpha_{n}=\infty, \varepsilon_{n}=o\left(\alpha_{n}\right)$, where $\varepsilon_{n}=o\left(\alpha_{n}\right)$ means $\lim _{n \rightarrow \infty} \frac{\varepsilon_{n}}{\alpha_{n}}=0$;

(ii) $\alpha_{n}+\beta_{n}+\gamma_{n}=1, \forall n \geq 1$ and $\liminf _{n \rightarrow \infty} \beta_{n} \gamma_{n}>0$;

(iii) either $\sum_{n=1}^{\infty}\left\|e_{n}\right\|<\infty$ or $\lim _{n \rightarrow \infty} \frac{\left\|e_{n}\right\|}{\alpha_{n}}=0$;

(iv) $\theta \in[0,1), 0 \leq \theta_{n} \leq \bar{\theta}_{n}$, where

$$
\bar{\theta}_{n}=\left\{\begin{array}{l}
\min \left\{\theta, \frac{\varepsilon_{n}}{\left\|x_{n}-x_{n-1}\right\|}\right\}, x_{n} \neq x_{n-1}, \\
\theta, \text { otherwise. }
\end{array}\right.
$$

Then $\left\{x_{n}\right\}$ converges strongly to $z=P_{F(T)} x_{0}$. 
It is our purpose in this paper to extend the strong convergence result of this Halpern-type algorithm with both inertial and error terms to the class of strictly pseudocontractive mappings. Our strong convergence theorems extend the corresponding convergence theorems of [7] for nonexpansive mappings to the much more general class of strictly pseudocontractive mappings. Strong convegence of the algorithm is also proved for the iterative approximation of zeros of inverse strongly monotone operators. Implementation of our algorithm is illustrated by numerical examples in both finite and infinite dimensional real Hilbert spaces.

\section{PRELIMinARIES}

We need the following results to prove our main convergence theorem.

Lemma 2.1. Let $H$ be a real Hilbert space. Then, the following equalities and inequalities hold:

(i) $\|x+y\|^{2}=\|x\|^{2}+2\langle x, y\rangle+\|y\|^{2}, \forall x, y \in H$.

(ii) $\|x+y\|^{2} \leq\|x\|^{2}+2\langle y, x+y\rangle, \forall x, y \in H$.

(iii) $\|\alpha x+\beta y\|^{2}=\alpha(\alpha+\beta)\|x\|^{2}+\beta(\alpha+\beta)\|y\|^{2}-\alpha \beta\|x-y\|^{2}, \forall x, y \in H ; \forall \alpha, \beta \in \mathbb{R}$.

(iv) $\|\alpha x+\beta y+\gamma z\|^{2}=\alpha\|x\|^{2}+\beta\|y\|^{2}+\gamma\|z\|^{2}-\alpha \beta\|x-y\|^{2}-\alpha \gamma\|x-z\|^{2}-\beta \gamma\|y-z\|^{2}, \forall x, y \in$ $H ; \forall \alpha, \beta, \gamma \in[0,1], \alpha+\beta+\gamma=1$.

Lemma 2.2. ([16]) Let $C$ be a nonempty closed convex subset of a real Hilbert space $H$ and let $T: C \rightarrow C$ be a $k$-strictly pseudocontractive mapping. Then

(i) $(I-T)$ is demiclosed at 0, i.e, if $x_{n} \rightarrow x \in C$ and $x_{n}-T x_{n} \rightarrow 0$, then $x=T x$.

(ii) $F(T)=\{x \in C: T x=x\}$ is closed and convex.

Lemma 2.3. ([17]) Let $\left\{\Gamma_{n}\right\}$ be a sequence of real numbers. Assume $\left\{\Gamma_{n}\right\}$ does not decrease at infinity, that is, there exists at least a subsequence $\left\{\Gamma_{n_{k}}\right\}$ of $\left\{\Gamma_{n}\right\}$ such that $\Gamma_{n_{k}} \leq \Gamma_{n_{k}+1}$ for all $k \geq 0$. For every $n \geq n_{0}$, define an integer sequence $\{\tau(n)\}$ as

$$
\tau(n)=\max \left\{k \leq n: \Gamma_{k} \leq \Gamma_{k+1}\right\} .
$$

Then $\tau(n) \rightarrow \infty$ as $n \rightarrow \infty$, and, for all $n \geq n_{0}, \max \left\{\Gamma_{\tau(n)}, \Gamma_{n}\right\} \leq \Gamma_{\tau(n)+1}$.

Lemma 2.4. ([18]) Let $\left\{a_{n}\right\}_{n=1}^{\infty},\left\{c_{n}\right\}_{n=1}^{\infty},\left\{e_{n}\right\}_{n=1}^{\infty} \subset \mathbb{R}^{+}=[0, \infty),\left\{b_{n}\right\}_{n=1}^{\infty} \subset(0,1)$ and $\left\{d_{n}\right\}_{n=1}^{\infty} \subset \mathbb{R}$ be sequences such that

$$
a_{n+1} \leq\left[1-b_{n}+c_{n}\right] a_{n}+d_{n}+e_{n}, n \geq 1 .
$$

If $\sum_{n=1}^{\infty} c_{n}<\infty$ and $\sum_{n=1}^{\infty} e_{n}<\infty$, then

(i) If $d_{n} \leq M b_{n}$ for some $M \geq 0$, then $\left\{a_{n}\right\}_{n=1}^{\infty}$ is bounded.

(ii) If $\lim _{n \rightarrow \infty} b_{n}=0 ; \sum_{n=1}^{\infty} b_{n}=\infty$, and $\lim \sup _{n \rightarrow \infty} \frac{d_{n}}{b_{n}} \leq 0$, then $\lim _{n \rightarrow \infty} a_{n}=0$.

\section{Main Results}

Theorem 3.1. Let $H$ be a real Hilbert space and let $T: H \rightarrow H$ be a $k$-strictly pseudocontractive mapping with a nonempty fixed point set $F(T)$. Let $\left\{x_{n}\right\}$ be the sequence generated from arbitrary $x_{0}, x_{1} \in H$ by

$$
\left\{\begin{array}{l}
y_{n}=x_{n}+\theta_{n}\left(x_{n}-x_{n-1}\right), n \geq 1, \\
x_{n+1}=\alpha_{n} x_{0}+\beta_{n} y_{n}+\gamma_{n} T y_{n}+e_{n}, n \geq 1,
\end{array}\right.
$$


where $\left\{\alpha_{n}\right\},\left\{\beta_{n}\right\},\left\{\gamma_{n}\right\}$ are sequences in $(0,1),\left\{\varepsilon_{n}\right\}$ is a positive sequence and $\left\{e_{n}\right\} \subseteq H$ is a sequence of errors satisfying the conditions:

(i) $\lim _{n \rightarrow \infty} \alpha_{n}=0, \sum_{n=1}^{\infty} \alpha_{n}=\infty, \varepsilon_{n}=o\left(\alpha_{n}\right)$, where $\varepsilon_{n}=o\left(\alpha_{n}\right)$ means $\lim _{n \rightarrow \infty} \frac{\varepsilon_{n}}{\alpha_{n}}=0$;

(ii) $\alpha_{n}+\beta_{n}+\gamma_{n}=1, \forall n \geq 1, \beta_{n} \geq k, \forall n \geq 1$ and $\liminf _{n \rightarrow \infty} \gamma_{n}\left[\beta_{n}-\left(1-\alpha_{n}\right) k\right]>0$;

(iii) either $\sum_{n=1}^{\infty}\left\|e_{n}\right\|<\infty$ or $\lim _{n \rightarrow \infty} \frac{\left\|e_{n}\right\|}{\alpha_{n}}=0$;

(iv) $\theta \in[0,1), 0 \leq \theta_{n} \leq \bar{\theta}_{n}$, where

$$
\overline{\theta_{n}}=\left\{\begin{array}{l}
\min \left\{\theta, \frac{\varepsilon_{n}}{\left\|x_{n}-x_{n-1}\right\|}\right\}, x_{n} \neq x_{n-1}, \\
\theta, \text { otherwise. }
\end{array}\right.
$$

Then, Algorithm (3.1) converges strongly to $z=P_{F(T)} x_{0}$.

Proof. Let $p \in F(T)$. Using Lemma 2.1 and the properties of $T$, we obtain

$$
\begin{aligned}
\left\|x_{n+1}-p\right\|^{2}= & \left\|\alpha_{n}\left(x_{0}-p\right)+\beta_{n}\left(y_{n}-p\right)+\gamma_{n}\left(T y_{n}-p\right)\right\|^{2}+\left\|e_{n}\right\|^{2} \\
& +2\left\langle e_{n}, \alpha_{n}\left(x_{0}-p\right)+\beta_{n}\left(y_{n}-p\right)+\gamma_{n}\left(T y_{n}-p\right)\right\rangle \\
\leq & \alpha_{n}\left\|x_{0}-p\right\|^{2}+\beta_{n}\left\|y_{n}-p\right\|^{2}+\gamma_{n}\left\|T y_{n}-p\right\|^{2}+\left\|e_{n}\right\|^{2} \\
& -\alpha_{n} \beta_{n}\left\|x_{0}-y_{n}\right\|^{2}-\alpha_{n} \gamma_{n}\left\|x_{0}-T y_{n}\right\|^{2}-\beta_{n} \gamma_{n}\left\|y_{n}-T y_{n}\right\|^{2} \\
& +2\left\|e_{n}\right\|\left[\alpha_{n}\left\|x_{0}-p\right\|+\beta_{n}\left\|y_{n}-p\right\|+\gamma_{n}\left\|T y_{n}-p\right\|\right] \\
\leq & {\left[\beta_{n}+\gamma_{n}\right]\left\|y_{n}-p\right\|^{2}+\alpha_{n}\left\|x_{0}-p\right\|^{2}-\gamma_{n}\left[\beta_{n}-k\right]\left\|y_{n}-T y_{n}\right\|^{2} } \\
& -\alpha_{n} \beta_{n}\left\|x_{0}-y_{n}\right\|^{2}-\alpha_{n} \gamma_{n}\left\|T y_{n}-x_{0}\right\|^{2}+\left\|e_{n}\right\|^{2} \\
& +2 \alpha_{n}\left\|e_{n}\right\|\left\|x_{0}-p\right\|+2\left(\beta_{n}+\gamma_{n} L\right)\left\|e_{n}\right\|\left\|y_{n}-p\right\| \\
\leq & {\left[\left(\beta_{n}+\gamma_{n}\right)+2\left(\beta_{n}+\gamma_{n} L\right)\left\|e_{n}\right\|\right]\left\|y_{n}-p\right\|^{2}+\alpha_{n}\left\|x_{0}-p\right\|^{2}+2 \alpha_{n}\left\|e_{n}\right\|\left\|x_{0}-p\right\| } \\
& +\left\|e_{n}\right\|^{2}-\gamma_{n}\left[\beta_{n}-k\right]\left\|y_{n}-T y_{n}\right\|^{2}-\alpha_{n} \beta_{n}\left\|y_{n}-x_{0}\right\|^{2} \\
& -\alpha_{n} \gamma_{n}\left\|T y_{n}-x_{0}\right\|^{2}+2\left(\beta_{n}+\gamma_{n} L\right)\left\|e_{n}\right\| .
\end{aligned}
$$

Furthermore,

$$
\begin{aligned}
\left\|y_{n}-p\right\|^{2} & =\left\|x_{n}-p\right\|^{2}+\theta_{n}^{2}\left\|x_{n}-x_{n-1}\right\|^{2}+2\left\langle\theta_{n}\left(x_{n}-x_{n-1}\right), x_{n}-p\right\rangle \\
& \leq\left\|x_{n}-p\right\|^{2}+\theta_{n}^{2}\left\|x_{n}-x_{n-1}\right\|^{2}+2 \theta\left\|x_{n}-x_{n-1}\right\|\left\|x_{n}-p\right\| \\
& \leq\left\|x_{n}-p\right\|^{2}+\theta_{n}^{2}\left\|x_{n}-x_{n-1}\right\|^{2}+2 \theta_{n}\left\|x_{n}-x_{n-1}\right\|\left\|x_{n}-p\right\|^{2}+2 \theta_{n}\left\|x_{n}-x_{n-1}\right\| \\
& =\left[1+2 \theta_{n}\left\|x_{n}-x_{n-1}\right\|\right]\left\|x_{n}-p\right\|^{2}+\theta_{n}^{2}\left\|x_{n}-x_{n-1}\right\|^{2}+2 \theta_{n}\left\|x_{n}-x_{n-1}\right\| .
\end{aligned}
$$

Substituting (3.3) into (3.2), we obtain

$$
\begin{aligned}
\left\|x_{n+1}-p\right\|^{2} \leq & {\left[\left(\beta_{n}+\gamma_{n}\right)+2\left(\beta_{n}+\gamma_{n} L\right)\left\|e_{n}\right\|\right]\left\{\left[1+2 \theta_{n}\left\|x_{n}-x_{n-1}\right\|\right]\left\|x_{n}-p\right\|^{2}\right.} \\
& \left.+\theta_{n}^{2}\left\|x_{n}-x_{n-1}\right\|^{2}+2 \theta_{n}\left\|x_{n}-x_{n-1}\right\|\right\}+\alpha_{n}\left\|x_{0}-p\right\|^{2}+2 \alpha_{n}\left\|e_{n}\right\|\left\|x_{0}-p\right\| \\
& +\left\|e_{n}\right\|^{2}-\gamma_{n}\left[\beta_{n}-k\right]\left\|y_{n}-T y_{n}\right\|^{2}-\alpha_{n} \beta_{n}\left\|y_{n}-x_{0}\right\|^{2} \\
& -\alpha_{n} \gamma_{n}\left\|T y_{n}-x_{0}\right\|^{2}+2\left(\beta_{n}+\gamma_{n} L\right)\left\|e_{n}\right\| \\
\leq & {\left[1-\alpha_{n}+2\left(\beta_{n}+\gamma_{n}\right) \theta_{n}\left\|x_{n}-x_{n-1}\right\|\right.} \\
& \left.+2\left(\beta_{n}+\gamma_{n} L\right)\left\|e_{n}\right\|\left(1+2 \theta_{n}\left\|x_{n}-x_{n-1}\right\|\right)\right]\left\|x_{n}-p\right\|^{2} \\
& +\left[\left(\beta_{n}+\gamma_{n}\right)+2\left(\beta_{n}+L \gamma_{n}\right)\left\|e_{n}\right\|\right]\left[\theta_{n}^{2}\left\|x_{n}-x_{n-1}\right\|^{2}+2 \theta_{n}\left\|x_{n}-x_{n-1}\right\|\right] \\
& +\alpha_{n}\left\|x_{0}-p\right\|^{2}+2 \alpha_{n}\left\|e_{n}\right\|\left\|x_{0}-p\right\|+\left\|e_{n}\right\|^{2}+2\left(\beta_{n}+\gamma_{n} L\right)\left\|e_{n}\right\| .
\end{aligned}
$$


If $\lim _{n \rightarrow \infty} \frac{\left\|e_{n}\right\|}{\alpha_{n}}=0$, we can express (3.4) as follows

$$
\begin{aligned}
\left\|x_{n+1}-p\right\|^{2} \leq & {\left[1-\alpha_{n}+\alpha_{n}\left\{2\left(\beta_{n}+\gamma_{n}\right) \frac{\theta_{n}}{\alpha_{n}}\left\|x_{n}-x_{n-1}\right\|\right.\right.} \\
& \left.\left.+2 \frac{\left\|e_{n}\right\|}{\alpha_{n}}\left(\beta_{n}+\gamma_{n} L\right)\left(1+2 \theta_{n}\left\|x_{n}-x_{n-1}\right\|\right)\right\}\right]\left\|x_{n}-p\right\|^{2} \\
& +\alpha_{n}\left\{[ ( \beta _ { n } + \gamma _ { n } ) + 2 \alpha _ { n } ( \beta _ { n } + \gamma _ { n } L ) \frac { \| e _ { n } \| } { \alpha _ { n } } ] \left[\alpha_{n}\left(\frac{\theta_{n}}{\alpha_{n}}\right)^{2}\left\|x_{n}-x_{n-1}\right\|^{2}\right.\right. \\
& \left.+2 \frac{\theta_{n}}{\alpha_{n}}\left\|x_{n}-x_{n-1}\right\|\right]+\left\|x_{0}-p\right\|^{2}+2 \alpha_{n} \frac{\left\|e_{n}\right\|}{\alpha_{n}}\left\|x_{0}-p\right\| \\
& \left.+\alpha_{n}\left(\frac{\left\|e_{n}\right\|}{\alpha_{n}}\right)^{2}+2 \frac{\left\|e_{n}\right\|}{\alpha_{n}}\left(\beta_{n}+\gamma_{n} L\right)\right\} .
\end{aligned}
$$

The hypothesis of our Theorem imply that there exists $D>0$ such that

$$
\begin{aligned}
& 2\left\{[ ( \beta _ { n } + \gamma _ { n } ) + 2 \alpha _ { n } ( \beta _ { n } + \gamma _ { n } L ) \frac { \| e _ { n } \| } { \alpha _ { n } } ] \left[\alpha_{n}\left(\frac{\theta_{n}}{\alpha_{n}}\right)^{2}\left\|x_{n}-x_{n-1}\right\|^{2}\right.\right. \\
& \left.+2 \frac{\theta_{n}}{\alpha_{n}}\left\|x_{n}-x_{n-1}\right\|\right]+\left\|x_{0}-p\right\|^{2}+2 \alpha_{n} \frac{\left\|e_{n}\right\|}{\alpha_{n}}\left\|x_{0}-p\right\| \\
& \left.+\alpha_{n}\left(\frac{\left\|e_{n}\right\|}{\alpha_{n}}\right)^{2}+2 \frac{\left\|e_{n}\right\|}{\alpha_{n}}\left(\beta_{n}+\gamma_{n} L\right)\right\} \leq D, \forall n \geq 1
\end{aligned}
$$

Furthermore, there exists a positive integer $N$ such that

$$
\left\{2\left(\beta_{n}+\gamma_{n}\right) \frac{\theta_{n}}{\alpha_{n}}\left\|x_{n}-x_{n-1}\right\|+2 \frac{\left\|e_{n}\right\|}{\alpha_{n}}\left(\beta_{n}+\gamma_{n}\right)\left(1+2 \theta_{n}\left\|x_{n}-x_{n-1}\right\|\right)\right\} \leq \frac{1}{2}, \quad \forall n \geq N .
$$

Thus, substituting (3.6) and (3.7) into (3.5), we obtain

$$
\left\|x_{n+1}-p\right\|^{2} \leq\left[1-\sigma_{n}\right]\left\|x_{n}-p\right\|^{2}+D \sigma_{n}, n \geq N,
$$

where $\sigma_{n}=\frac{\alpha_{n}}{2}$. It now follows from Lemma 2.4 that $\left\{x_{n}\right\}$ is bounded. If $\sum_{n=1}^{\infty}\left\|e_{n}\right\|<\infty$, let $N$ be a positive integer such that

$$
2 \frac{\theta_{n}}{\alpha_{n}}\left(\beta_{n}+\gamma_{n}\right)\left\|x_{n}-x_{n-1}\right\| \leq \frac{1}{2}, \forall n \geq N
$$

Furtherfore, let $M_{1}>0, M_{2}>0$ and $M_{3}>0$ be such that

$$
\begin{aligned}
& 2\left(\beta_{n}+\gamma_{n}\right)\left(1+2 \theta_{n}\left\|x_{n}-x_{n-1}\right\|\right) \leq M_{1}, \forall n \geq 1, \\
& 2\left(\beta_{n}+\gamma_{n}\right)\left[\alpha_{n}\left(\frac{\theta_{n}}{\alpha_{n}}\right)^{2}\left\|x_{n}-x_{n-1}\right\|^{2}+2 \frac{\theta_{n}}{\alpha_{n}}\left\|x_{n}-x_{n-1}\right\|\right] \\
& +\left\|x_{0}-p\right\|^{2}+2\left\|e_{n}\right\|\left\|x_{0}-p\right\| \leq M_{2}, \forall n \geq 1
\end{aligned}
$$

and

$$
\left[2\left(\beta_{n}+\gamma_{n} L\right)\left(\theta_{n}^{2}\left\|x_{n}-x_{n-1}\right\|^{2}+2 \theta_{n}\left\|x_{n}-x_{n-1}\right\|+1\right)+\left\|e_{n}\right\|\right]\left\|e_{n}\right\| \leq M_{3}, \forall n \geq 1
$$


From (3.4), we obtain

$$
\begin{aligned}
& \left\|x_{n+1}-p\right\|^{2} \\
& \leq\left[1-\alpha_{n}+\alpha_{n}\left(2 \frac{\theta_{n}}{\alpha_{n}}\left(\beta_{n}+\gamma_{n}\right)\left\|x_{n}-x_{n-1}\right\|\right)\right. \\
& \left.\quad+2\left(\beta_{n}+\gamma_{n}\right)\left(1+2 \theta_{n}\left\|x_{n}-x_{n-1}\right\|\right)\left\|e_{n}\right\|\right]\left\|x_{n}-p\right\|^{2} \\
& \quad+\alpha_{n}\left(2\left(\beta_{n}+\gamma_{n}\right)\left[\alpha_{n}\left(\frac{\theta_{n}}{\alpha_{n}}\right)^{2}\left\|x_{n}-x_{n-1}\right\|^{2}+2 \frac{\theta_{n}}{\alpha_{n}}\left\|x_{n}-x_{n-1}\right\|\right]\right. \\
& \quad+\left\|x_{0}-p\right\|^{2}+2\left\|e_{n}\right\|\left\|x_{0}-p\right\| \\
& \quad+\left[2\left(\beta_{n}+\gamma_{n} L\right)\left(\theta_{n}^{2}\left\|x_{n}-x_{n-1}\right\|^{2}+2 \theta_{n}\left\|x_{n}-x_{n-1}\right\|+1\right)+\left\|e_{n}\right\|\right]\left\|e_{n}\right\|, \forall n \geq N .
\end{aligned}
$$

Thus,

$$
\begin{aligned}
\left\|x_{n+1}-p\right\|^{2} & \leq\left[1-\frac{\alpha_{n}}{2}+M_{1}\left\|e_{n}\right\|\right]\left\|x_{n}-p\right\|^{2}+M_{2} \frac{\alpha_{n}}{2}+M_{3}\left\|e_{n}\right\| \\
& =\left[1-\sigma_{n}+\delta_{n}\right]\left\|x_{n}-p\right\|^{2}+M_{2} \sigma_{n}+\lambda_{n}, \forall n \geq N
\end{aligned}
$$

where $\sigma_{n}=\frac{\alpha_{n}}{2}, \delta_{n}=M_{1}\left\|e_{n}\right\|$, and $\lambda_{n}=M_{3}\left\|e_{n}\right\|$. It now follows from Lemma 2.4 that $\left\{x_{n}\right\}$ is bounded. Furthermore,

$$
\begin{aligned}
\left\|y_{n}-p\right\|^{2} & =\left\|x_{n}+\theta_{n}\left(x_{n}-x_{n-1}\right)-p\right\|^{2} \\
& =\left\|x_{n}-p\right\|^{2}+2 \theta_{n}\left\langle x_{n}-x_{n-1}, x_{n}-p\right\rangle+\theta_{n}^{2}\left\|x_{n}-x_{n-1}\right\|^{2} \\
& \leq\left\|x_{n}-p\right\|^{2}+2 \theta_{n}\left\langle x_{n}-x_{n-1}, x_{n}-p\right\rangle+\theta_{n}\left\|x_{n}-x_{n-1}\right\|^{2} .
\end{aligned}
$$

Observe that

$$
\begin{aligned}
\left\|x_{n-1}-p\right\|^{2} & =\left\|x_{n-1}-x_{n}+x_{n}-p\right\|^{2} \\
& =\left\|x_{n}-p\right\|^{2}-2\left\langle x_{n}-x_{n-1}, x_{n}-p\right\rangle+\left\|x_{n}-x_{n-1}\right\|^{2},
\end{aligned}
$$

which implies

$$
2\left\langle x_{n}-x_{n-1}, x_{n}-p\right\rangle=-\left\|x_{n-1}-p\right\|^{2}+\left\|x_{n}-p\right\|^{2}+\left\|x_{n}-x_{n-1}\right\|^{2} .
$$

Substituting (3.9) into (3.8), we obtain

$$
\begin{aligned}
\left\|y_{n}-p\right\|^{2} \leq & \left\|x_{n}-p\right\|^{2}+\theta_{n}\left[-\left\|x_{n-1}-p\right\|^{2}\right. \\
& \left.+\left\|x_{n}-p\right\|^{2}+\left\|x_{n}-x_{n-1}\right\|^{2}\right]+\theta_{n}\left\|x_{n}-x_{n-1}\right\|^{2} \\
= & \left\|x_{n}-p\right\|^{2}+2 \theta_{n}\left\|x_{n}-x_{n-1}\right\|^{2} \\
& +\theta_{n}\left[\left\|x_{n}-p\right\|^{2}-\left\|x_{n-1}-p\right\|^{2}\right] .
\end{aligned}
$$


On the other hand, we have

$$
\begin{aligned}
\left\|x_{n+1}-p\right\|^{2}= & \left\|\alpha_{n} x_{0}+\beta_{n} y_{n}+\gamma_{n} T y_{n}+e_{n}-p\right\|^{2} \\
= & \left\|\alpha_{n}\left(x_{0}-p+\frac{e_{n}}{\alpha_{n}}\right)+\beta_{n}\left(y_{n}-p\right)+\gamma_{n}\left(T y_{n}-p\right)\right\|^{2} \\
\leq & \left\|\beta_{n}\left(y_{n}-p\right)+\gamma_{n}\left(T y_{n}-p\right)\right\|^{2}+2\left\langle\alpha_{n}\left(x_{0}-p+\frac{e_{n}}{\alpha_{n}}\right), x_{n+1}-p\right\rangle \\
= & \beta_{n}\left(\beta_{n}+\gamma_{n}\right)\left\|y_{n}-p\right\|^{2}+\gamma_{n}\left(\beta_{n}+\gamma_{n}\right)\left\|T y_{n}-p\right\|^{2}-\beta_{n} \gamma_{n}\left\|y_{n}-T y_{n}\right\|^{2} \\
& +2\left\langle\alpha_{n}\left(x_{0}-p+\frac{e_{n}}{\alpha_{n}}\right), x_{n+1}-p\right\rangle \\
\leq & \beta_{n}\left(\beta_{n}+\gamma_{n}\right)\left\|y_{n}-p\right\|^{2}+\gamma_{n}\left(\beta_{n}+\gamma_{n}\right)\left[\left\|y_{n}-p\right\|^{2}+k\left\|y_{n}-T y_{n}\right\|^{2}\right] \\
& -\beta_{n} \gamma_{n}\left\|y_{n}-T y_{n}\right\|^{2}+2\left\langle\alpha_{n}\left(x_{0}-p+\frac{e_{n}}{\alpha_{n}}\right), x_{n+1}-p\right\rangle \\
= & \left(\beta_{n}+\gamma_{n}\right)^{2}\left\|y_{n}-p\right\|^{2}-\gamma_{n}\left[\beta_{n}-\left(\beta_{n}+\gamma_{n}\right) k\right]\left\|y_{n}-T y_{n}\right\|^{2} \\
& +2\left\langle\alpha_{n}\left(x_{0}-p+\frac{e_{n}}{\alpha_{n}}\right), x_{n+1}-p\right\rangle \\
\leq & \left(1-\alpha_{n}\right)\left\|y_{n}-p\right\|^{2}-\gamma_{n}\left[\beta_{n}-\left(1-\alpha_{n}\right) k\right]\left\|y_{n}-T y_{n}\right\|^{2} \\
& +2\left\langle\alpha_{n}\left(x_{0}-p+\frac{e_{n}}{\alpha_{n}}\right), x_{n+1}-p\right\rangle .
\end{aligned}
$$

Using (3.10) in (3.10), we obtain

$$
\begin{aligned}
\left\|x_{n+1}-p\right\|^{2} \leq & \left(1-\alpha_{n}\right)\left[\left\|x_{n}-p\right\|^{2}+2 \theta_{n}\left\|x_{n}-x_{n-1}\right\|^{2}+\theta_{n}\left[\left\|x_{n}-p\right\|^{2}-\left\|x_{n-1}-p\right\|^{2}\right]\right] \\
& -\gamma_{n}\left[\beta_{n}-\left(1-\alpha_{n}\right) k\right]\left\|y_{n}-T y_{n}\right\|^{2}+2\left\langle\alpha_{n}\left(x_{0}-p+\frac{e_{n}}{\alpha_{n}}\right), x_{n+1}-p\right\rangle \\
= & \left(1-\alpha_{n}\right)\left\|x_{n}-p\right\|^{2}-\gamma_{n}\left[\beta_{n}-\left(1-\alpha_{n}\right) k\right]\left\|y_{n}-T y_{n}\right\|^{2} \\
& +2 \theta_{n}\left(1-\alpha_{n}\right)\left\|x_{n}-x_{n-1}\right\|^{2}+\theta_{n}\left(1-\alpha_{n}\right)\left[\left\|x_{n}-p\right\|^{2}-\left\|x_{n-1}-p\right\|^{2}\right] \\
& +2 \alpha_{n}\left\langle x_{0}-p+\frac{e_{n}}{\alpha_{n}}, x_{n+1}-p\right\rangle .
\end{aligned}
$$

Set $\Gamma_{n}=\left\|x_{n}-p\right\|^{2}, \forall n \geq 1$. It follows from (3.11) that

$$
\begin{aligned}
\Gamma_{n+1} \leq & \left(1-\alpha_{n}\right) \Gamma_{n}-\gamma_{n}\left[\beta_{n}-\left(1-\alpha_{n}\right) k\right]\left\|y_{n}-T y_{n}\right\|^{2} \\
& +\theta_{n}\left(1-\alpha_{n}\right)\left(\Gamma_{n}-\Gamma_{n-1}\right)+2 \theta_{n}\left(1-\alpha_{n}\right)\left\|x_{n}-x_{n-1}\right\|^{2} \\
& +2 \alpha_{n}\left\langle x_{0}-p+\frac{e_{n}}{\alpha_{n}}, x_{n+1}-p\right\rangle .
\end{aligned}
$$

We consider two cases.

Case 1. Suppose that there is $N \in \mathbb{N}$ such that $\Gamma_{n+1} \leq \Gamma_{n}, \forall n \geq N$. Then $\lim _{n \rightarrow \infty} \Gamma_{n}$ exists and it follows from (3.12) and the hypothesis of the theorem that $\lim _{n \rightarrow \infty}\left\|y_{n}-T y_{n}\right\|=0$. Thus, from the hypothesis of the theorem, Lemma 2.2, and Lemma 2.3, some standard arguments (see for example $[17,15])$ yield that $\left\{x_{n}\right\}$ converges strongly to $z=P_{F}(T) x_{0}$.

Case 2. Suppose that there is no $N \in \mathbb{N}$ such that $\left\{\Gamma_{n}\right\}_{n=N}^{\infty}$ is monotone decreasing. We again follow the techniques presented in $([17,15])$ to obtain that $\left\{x_{n}\right\}$ converges strongly to $z=$ $P_{F}(T) x_{0}$.

If $T$ is demicontractive, then we have the following result. 
Corollary 3.1. Let $H$ be a real Hilbert space. Let $T: H \rightarrow H$ be a demicontractive mapping with constant $k \in[0,1)$ and let $(I-T)$ be demiclosed at zero. Let $\left\{x_{n}\right\}$ be the sequence generated from arbitrary $x_{0}, x_{1} \in H$ by Algorithm (3.1):

$$
\left\{\begin{array}{l}
y_{n}=x_{n}+\theta_{n}\left(x_{n}-x_{n-1}\right), n \geq 1, \\
x_{n+1}=\alpha_{n} x_{0}+\beta_{n} y_{n}+\gamma_{n} T y_{n}+e_{n}, n \geq 1,
\end{array}\right.
$$

where $\left\{\alpha_{n}\right\},\left\{\beta_{n}\right\},\left\{\gamma_{n}\right\}$ are sequences in $(0,1),\left\{\varepsilon_{n}\right\}$ is a positive sequence and $\left\{e_{n}\right\} \subseteq H$ is a sequence of errors and the sequences satisfy the conditions:

(i) $\lim _{n \rightarrow \infty} \alpha_{n}=0, \sum_{n=1}^{\infty} \alpha_{n}=\infty, \varepsilon_{n}=o\left(\alpha_{n}\right)$, where $\varepsilon_{n}=o\left(\alpha_{n}\right)$ means $\lim _{n \rightarrow \infty} \frac{\varepsilon_{n}}{\alpha_{n}}=0$;

(ii) $\alpha_{n}+\beta_{n}+\gamma_{n}=1, \forall n \geq 1, \beta_{n} \geq k, \forall n \geq 1$ and $\liminf _{n \rightarrow \infty} \gamma_{n}\left[\beta_{n}-\left(1-\alpha_{n}\right) k\right]>0$;

(iii) either $\sum_{n=1}^{\infty}\left\|e_{n}\right\|<\infty$ or $\lim _{n \rightarrow \infty} \frac{\left\|e_{n}\right\|}{\alpha_{n}}=0$;

(iv) $\theta \in[0,1), 0 \leq \theta_{n} \leq \bar{\theta}_{n}$, where

$$
\bar{\theta}_{n}=\left\{\begin{array}{l}
\min \left\{\theta, \frac{\varepsilon_{n}}{\left\|x_{n}-x_{n-1}\right\|}\right\}, x_{n} \neq x_{n-1}, \\
\theta, \text { otherwise. }
\end{array}\right.
$$

Then $\left\{x_{n}\right\}$ converges strongly to $z=P_{F(T)} x_{0}$.

Next, we give some real sequences, which satisfy the restrictions on the parameters: $\alpha_{n}=$ $\frac{1-k}{2(n+1)}, \beta_{n}=\frac{1+k}{2}+\frac{1-k}{2(n+1)}, \gamma_{n}=1-\frac{1+k}{2}-\frac{1-k}{n+1}, \varepsilon_{n}=\frac{1}{(n+1)^{2}}, e_{n}=\frac{c}{(n+1)^{2}}$, where $c \in H$ is any fixed vector.

Remark 3.1. In [16], Osilike and Udomene showed that if $T$ is $k$-strictly pseudocontractive, then the operator $T_{\beta}=(1-\beta) I+\beta T$ is nonexpansive for every $\beta \in(0,1-k]$.

Thus, the following theorems directly follow from the Theorem 1.1 and Theorem 4.4 of [15], respectively.

Corollary 3.2. Let $H$ be a real Hilbert space and let $T: H \rightarrow H$ be a $k$-strictly pseudocontractive mapping with a nonempty fixed point set $F(T)$. Let $\left\{x_{n}\right\}$ be the sequence generated from arbitrary $x_{0}, x_{1} \in H$ by

$$
\left\{\begin{array}{l}
y_{n}=x_{n}+\theta_{n}\left(x_{n}-x_{n-1}\right), n \geq 1 \\
x_{n+1}=\alpha_{n} x_{0}+\beta_{n} y_{n}+\gamma_{n} T_{\beta} y_{n}+e_{n}, n \geq 1
\end{array}\right.
$$

where $\left\{\alpha_{n}\right\},\left\{\beta_{n}\right\},\left\{\gamma_{n}\right\}$ are sequences in $(0,1),\left\{\varepsilon_{n}\right\}$ is a positive sequence and $\left\{e_{n}\right\} \subseteq H$ is a sequence of errors and the sequences satisfy the conditions:

(i) $\lim _{n \rightarrow \infty} \alpha_{n}=0, \sum_{n=1}^{\infty} \alpha_{n}=\infty, \varepsilon_{n}=o\left(\alpha_{n}\right)$, where $\varepsilon_{n}=o\left(\alpha_{n}\right)$ means $\lim _{n \rightarrow \infty} \frac{\varepsilon_{n}}{\alpha_{n}}=0$;

(ii) $\alpha_{n}+\beta_{n}+\gamma_{n}=1, \forall n \geq 1$ and $\liminf _{n \rightarrow \infty} \beta_{n} \gamma_{n}>0$;

(iii) either $\sum_{n=1}^{\infty}\left\|e_{n}\right\|<\infty$ or $\lim _{n \rightarrow \infty} \frac{\left\|e_{n}\right\|}{\alpha_{n}}=0$;

(iv) $\theta \in[0,1), 0 \leq \theta_{n} \leq \bar{\theta}_{n}$, where

$$
\bar{\theta}_{n}=\left\{\begin{array}{l}
\min \left\{\theta, \frac{\varepsilon_{n}}{\left\|x_{n}-x_{n-1}\right\|}\right\}, x_{n} \neq x_{n-1}, \\
\theta, \text { otherwise. }
\end{array}\right.
$$

Then, Algorithm (3.14) converges strongly to $z=P_{F(T)} x_{0}$. 
Corollary 3.3. Let $H$ be a real Hilbert space and let $T: H \rightarrow H$ be a demicontractive mapping with constant $k \in[0,1)$ and let $(I-T)$ be demiclosed at zero. Let $\left\{x_{n}\right\}$ be the sequence generated from arbitrary $x_{0}, x_{1} \in H$ by Algorithm (3.14):

$$
\left\{\begin{array}{l}
y_{n}=x_{n}+\theta_{n}\left(x_{n}-x_{n-1}\right), n \geq 1, \\
x_{n+1}=\alpha_{n} x_{0}+\beta_{n} y_{n}+\gamma_{n} T_{\beta} y_{n}+e_{n}, n \geq 1,
\end{array}\right.
$$

where $\left\{\alpha_{n}\right\},\left\{\beta_{n}\right\},\left\{\gamma_{n}\right\}$ are sequences in $(0,1),\left\{\varepsilon_{n}\right\}$ is a positive sequence and $\left\{e_{n}\right\} \subseteq H$ is a sequence of errors and the sequences satisfy the conditions:

(i) $\lim _{n \rightarrow \infty} \alpha_{n}=0, \sum_{n=1}^{\infty} \alpha_{n}=\infty, \varepsilon_{n}=o\left(\alpha_{n}\right)$, where $\varepsilon_{n}=o\left(\alpha_{n}\right)$ means $\lim _{n \rightarrow \infty} \frac{\varepsilon_{n}}{\alpha_{n}}=0$;

(ii) $\alpha_{n}+\beta_{n}+\gamma_{n}=1, \forall n \geq 1$, and $\liminf _{n \rightarrow \infty} \beta_{n} \gamma_{n}>0$;

(iii) either $\sum_{n=1}^{\infty}\left\|e_{n}\right\|<\infty$ or $\lim _{n \rightarrow \infty} \frac{\left\|e_{n}\right\|}{\alpha_{n}}=0$;

(iv) $\theta \in[0,1), 0 \leq \theta_{n} \leq \bar{\theta}_{n}$, where

$$
\bar{\theta}_{n}=\left\{\begin{array}{l}
\min \left\{\theta, \frac{\varepsilon_{n}}{\left\|x_{n}-x_{n-1}\right\|}\right\}, x_{n} \neq x_{n-1}, \\
\theta, \text { otherwise. }
\end{array}\right.
$$

Then, Algorithm (3.14) converges strongly to $z=P_{F(T)} x_{0}$.

We remark that Algorithm (3.1) takes less time to be implemented than Algorithm 3.14, which is implemented with an auxiliary operator (see our examples below).

Zeros of inverse-strongly monotone operators

Definition 3.1. An operator $A: H \rightarrow H$ is said to be inverse-strongly monotone if there exists $\alpha>0$ such that

$$
\langle A x-A y, x-y\rangle \geq \alpha\|A x-A y\|^{2}, \forall x, y \in H .
$$

It is also said to be $\alpha$-inverse-strongly monotone.

We may assume without loss of generality that $\alpha \in[0,1)$. It is easy to observe that if $A$ is $\alpha$-inverse-strongly monotone, then the operator $T=I-A$ is $(1-\alpha)$-strictly pseudocontractive. Thus we have the following.

Theorem 3.2. Let $H$ be a real Hilbert space, and let $A: H \rightarrow H$ be an $\alpha$-inverse-strongly monotone mapping with $A^{-1} 0=\{x \in H: A x=0\} \neq \emptyset$. Let $T=I-A$ and let $\left\{x_{n}\right\}$ be the sequence generated from arbitrary $x_{0}, x_{1} \in H$ by (3.1):

$$
\left\{\begin{array}{l}
y_{n}=x_{n}+\theta_{n}\left(x_{n}-x_{n-1}\right), n \geq 1, \\
x_{n+1}=\alpha_{n} x_{0}+\beta_{n} y_{n}+\gamma_{n} T y_{n}+e_{n}, n \geq 1,
\end{array}\right.
$$

where $\left\{\alpha_{n}\right\},\left\{\beta_{n}\right\},\left\{\gamma_{n}\right\}$ are sequences in $(0,1),\left\{\varepsilon_{n}\right\}$ is a positive sequence and $\left\{e_{n}\right\} \subseteq H$ is a sequence of errors and these sequences satisfy the conditions:

(i) $\lim _{n \rightarrow \infty} \alpha_{n}=0, \sum_{n=1}^{\infty} \alpha_{n}=\infty, \varepsilon_{n}=o\left(\alpha_{n}\right)$, where $\varepsilon_{n}=o\left(\alpha_{n}\right)$ means $\lim _{n \rightarrow \infty} \frac{\varepsilon_{n}}{\alpha_{n}}=0$;

(ii) $\alpha_{n}+\beta_{n}+\gamma_{n}=1, \forall n \geq 1, \beta_{n} \geq k, \forall n \geq 1$ and $\liminf _{n \rightarrow \infty} \gamma_{n}\left[\beta_{n}-\left(1-\alpha_{n}\right) k\right]>0, k=(1-\alpha) \in$ $[0,1)$;

(iii) either $\sum_{n=1}^{\infty}\left\|e_{n}\right\|<\infty$ or $\lim _{n \rightarrow \infty} \frac{\left\|e_{n}\right\|}{\alpha_{n}}=0$;

(iv) $\theta \in[0,1), 0 \leq \theta_{n} \leq \bar{\theta}_{n}$, where

$$
\bar{\theta}_{n}=\left\{\begin{array}{l}
\min \left\{\theta, \frac{\varepsilon_{n}}{\left\|x_{n}-x_{n-1}\right\|}\right\}, x_{n} \neq x_{n-1}, \\
\theta, \text { otherwise. }
\end{array}\right.
$$


Then, Algorithm (3.1) converges strongly to $z=P_{F(T)} x_{0}=P_{A^{-1} 0} x_{0}$.

Definition 3.2. An operator $A: D(A) \subseteq H \rightarrow 2^{H}$ is said to be monotone if

$$
\langle A x-A y, u-v\rangle \geq 0, \forall x, y \in D(A) ; u \in A x, v \in A y .
$$

$A$ is said to be maximal monotone if $A$ is monotone and the graph of $A, G(A)=\{(x, u): x \in$ $D(A), u \in A x\}$ is not properly contained in the graph of any other monotone operator. The resolvent of $A$ with index $\lambda>0$ is the mapping $J_{\lambda}^{A}: H \rightarrow H$ given by $J_{\lambda}^{A} x=(I+\lambda A)^{-1} x$.

It is well-known that $J_{\lambda}^{A}$ is single-valued, nonexpansive and 1-inverse-strongly monotone and if $A^{-1} 0=\{x \in D(A): 0 \in A x\} \neq \emptyset$, then $x \in A^{-1} 0$ if and only if $x \in F\left(J_{\lambda}^{A}\right)$. Furthermore, if $A$ and $B$ are monotone, then $x \in(A+B)^{-1} 0$ if and only if $x \in F\left(J_{\lambda}^{A}(I-\lambda B)\right)$ (see, for example, [19]).

Let $A: H \rightarrow H$ be an $\alpha$-inverse-strongly monotone operator and let $B: H \rightarrow 2^{H}$ be a maximal monotone operator. Then, for $0<\lambda \leq 2 \alpha$, we have that $J_{\lambda}^{B}(I-\lambda A)$ is nonexpansive and hence strictly pseudocontractive. Thus, we have the following result.

Theorem 3.3. Let $A: H \rightarrow H$ be $\alpha$-inverse strongly monotone operator, and let $B: H \rightarrow 2^{H}$ be a maximal monotone operator. Let $0<\lambda \leq 2 \alpha$ and let $\left\{x_{n}\right\}$ be the sequence generated from arbitrary $x_{0}, x_{1} \in H$ by (3.1):

$$
\left\{\begin{array}{l}
y_{n}=x_{n}+\theta_{n}\left(x_{n}-x_{n-1}\right), n \geq 1, \\
x_{n+1}=\alpha_{n} x_{0}+\beta_{n} y_{n}+\gamma_{n} T y_{n}+e_{n}, n \geq 1,
\end{array}\right.
$$

where $T=J_{\lambda}^{B}(I-\lambda A),\left\{\alpha_{n}\right\},\left\{\beta_{n}\right\},\left\{\gamma_{n}\right\}$ are sequences in $(0,1),\left\{\varepsilon_{n}\right\}$ is a positive sequence and $\left\{e_{n}\right\} \subseteq H$ is a sequence of errors and the sequences satisfy the conditions:

(i) $\lim _{n \rightarrow \infty} \alpha_{n}=0, \sum_{n=1}^{\infty} \alpha_{n}=\infty, \varepsilon_{n}=o\left(\alpha_{n}\right)$, where $\varepsilon_{n}=o\left(\alpha_{n}\right)$ means $\lim _{n \rightarrow \infty} \frac{\varepsilon_{n}}{\alpha_{n}}=0$;

(ii) $\alpha_{n}+\beta_{n}+\gamma_{n}=1, \forall n \geq 1, \beta_{n} \geq k, \forall n \geq 1$ and $\liminf _{n \rightarrow \infty} \gamma_{n}\left[\beta_{n}-\left(1-\alpha_{n}\right) k\right]>0, k \in[0,1)$;

(iii) either $\sum_{n=1}^{\infty}\left\|e_{n}\right\|<\infty$ or $\lim _{n \rightarrow \infty} \frac{\left\|e_{n}\right\|}{\alpha_{n}}=0$;

(iv) $\theta \in[0,1), 0 \leq \theta_{n} \leq \bar{\theta}_{n}$, where

$$
\overline{\theta_{n}}=\left\{\begin{array}{l}
\min \left\{\theta, \frac{\varepsilon_{n}}{\left\|x_{n}-x_{n-1}\right\|}\right\}, x_{n} \neq x_{n-1}, \\
\theta, \text { otherwise. }
\end{array}\right.
$$

Then, Algorithm (3.1) converges strongly to $z=P_{F(T)} x_{0}=P_{(A+B)^{-1} 0} x_{0}$.

\section{NUMERICAL EXAMPLES}

In this section, the numerical examples to demonstrate the convergence of the proposed Algorithms (3.1) and (3.14) discussed are given in the real Hilbert space setting. From the examples, we show graphically strong convergence results discussed in Section 3. All codes are written in MATLAB, and implemented using an HP Elitebook 6930p computer with Pentium(R) DUAL-CORE CPU T4200 with $2.00 \mathrm{~Hz}$ and 2GB RAM.

Example 4.1. Let $\mathbb{R}$ denote the reals with the usual norm and define $T: \mathbb{R} \rightarrow \mathbb{R}$ by

$$
T x=\left\{\begin{array}{l}
-3 x+1, x \in(-\infty, 0], \\
\frac{1}{2}(x+2), x \in(0, \infty) .
\end{array}\right.
$$


Then $T$ is $\frac{1}{2}-$ strictly pseudocontractive. Let $x_{0}=0, x_{1}=1, \alpha_{n}=\frac{1}{4(n+1)} ; \beta_{n}=\frac{3}{4}+\frac{1}{4(n+1)}$; $\gamma_{n}=\frac{1}{4}-\frac{1}{2(n+1)} ; \varepsilon_{n}=\frac{1}{(n+1)^{2}} ; e_{n}=\frac{1}{(n+1)^{2}}$ and $\theta=\frac{1}{2}$. Then the Algorithms (3.1) and (3.14) converge strongly to 2 . The results are shown in Figure 1 and Table 1 below.

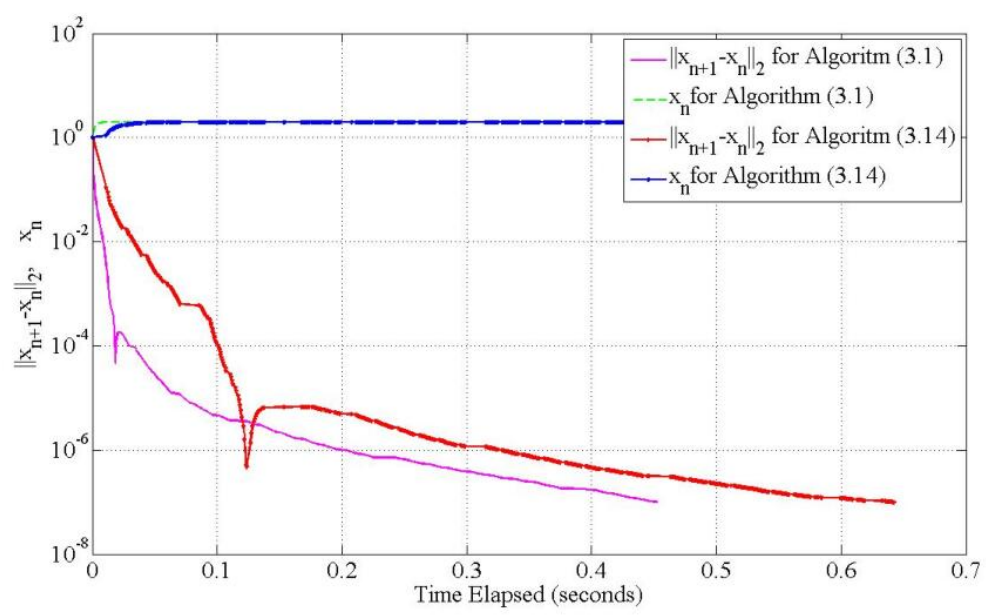

FIGURE 1. The convergence behaviors of the iterative algorithms

\begin{tabular}{|c|ccc||ccc|}
\hline $\begin{array}{c}\text { No. } \\
\text { of } \\
\text { iter. }\end{array}$ & \multicolumn{3}{|c||}{ Algorithm (3.1) } & \multicolumn{3}{c|}{ Algorithm (3.14) } \\
\hline $\mathrm{n}$ & Time & \multicolumn{3}{c||}{ Time } \\
& (Secs.) & $x_{n}$ & $\left\|x_{n+1}-x_{n}\right\|$ & (Secs.) & $x_{n}$ & $\left\|x_{n+1}-x_{n}\right\|$ \\
\hline 136 & 0.1075635 & 2.0002320 & 0.0000036 & 0.1244865 & 2.0005200 & 0.0000068 \\
246 & 0.2323397 & 2.0000690 & 0.0000006 & 0.2504222 & 2.0001500 & 0.0000013 \\
321 & 0.3039775 & 2.0000400 & 0.0000003 & 0.3346608 & 2.0000800 & 0.0000006 \\
435 & 0.4329729 & 2.0000220 & 0.0000001 & 0.4519594 & 2.0000400 & 0.0000002 \\
556 & 0.0000000 & 0.0000000 & 0.0000000 & 0.5695135 & 2.0000300 & 0.0000001 \\
\hline
\end{tabular}

TABLE 1. The numerical values of $\left\|x_{n+1}-x_{n}\right\|$ for the two algorithms compared

Example 4.2. Let $X=\ell_{2}(\mathbb{R})=\left\{x=\left\{x_{i}\right\}_{i=1}^{\infty}: x_{i} \in \mathbb{R}\right.$ and $\left.\sum_{i=1}^{\infty}\left|x_{i}\right|^{2}<\infty\right\}$. Define $T: X \rightarrow X$ by $T x=-3 x$. Then $T$ is $\frac{1}{2}$-strictly pseudocontractive. Let $x_{0}=(1,0,0,0, \ldots) ; x_{1}=\left(\frac{1}{2}, 0,0,0, \ldots\right)$, $\alpha_{n}=\frac{1}{4(n+1)} ; \beta_{n}=\frac{3}{4}+\frac{1}{4(n+1)} ; \gamma_{n}=\frac{1}{4}-\frac{1}{2(n+1)} ; \varepsilon_{n}=\frac{1}{(n+1)^{2}} ; \theta=\frac{1}{2}$ and $e_{n}=\frac{c}{(n+1)^{2}}$, where $c=$ $(1,0,0,0, \ldots)$. Then the Algorithms (3.1) and (3.14) converge strongly to $0=(0,0,0, \ldots)$ as in Figure 2 and Table 2 below

Remark 4.1. The stopping criteria is $\left\|x_{n+1}-x_{n}\right\| \leq 10^{-7}$ for both Tables which imply that the error of approximating the fixed point of the given mapping is negligible. From Figure 1 and Table 1, as the consecutive terms of the sequence get close enough (as close as a difference of $10^{-7}$ ), the sequence generated is seen to approach the fixed point of the mapping, which 


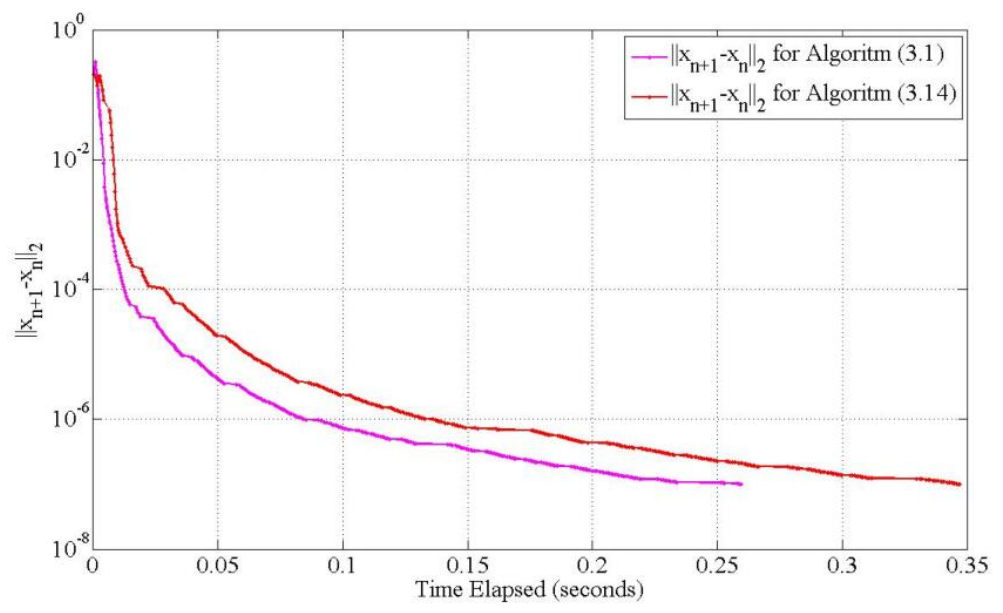

FIGURE 2. The convergence behaviors of the iterative algorithms

\begin{tabular}{|c|cc||cc|}
\hline $\begin{array}{c}\text { No. } \\
\text { of } \\
\text { iter. }\end{array}$ & Algorithm (3.1) & & \multicolumn{2}{||}{ Algorithm (3.14) } \\
\hline $\mathrm{n}$ & Time & & Time & \\
& (Secs.) & $\left\|x_{n+1}-x_{n}\right\|$ & (Secs.) & $\left\|x_{n+1}-x_{n}\right\|$ \\
\hline 21 & 0.0123282 & 0.0002369 & 0.0115707 & 0.0005230 \\
104 & 0.0686563 & 0.0000018 & 0.0782150 & 0.0000037 \\
215 & 0.1793904 & 0.0000002 & 0.1860124 & 0.0000004 \\
271 & 0.2432082 & 0.0000001 & 0.2530258 & 0.0000002 \\
343 & 0.0000000 & 0.0000000 & 0.3182514 & 0.0000001 \\
\hline
\end{tabular}

TABLE 2. The numerical values of $\left\|x_{n+1}-x_{n}\right\|$ for the two algorithms compared

is 2. A lower stopping criteria will make no much difference while a higher stopping criteria will truncate the computation too early which might lead to higher computational error. Clearly error $=\left\|x_{n}-2\right\| \rightarrow 0$ as $n \rightarrow \infty$, which shows that the algorithm converges to the desired fixed point. The same holds for Figure 2 and Table 2.

\section{CONCLUSiON}

A Halpern-type algorithm with both inertial and error terms for approximating fixed points of strictly pseudocontractive mappings and zeros of inverse-strongly monotone operators in real Hilbert spaces was discussed in this paper. Strong convergence results were proved for a $k$-strictly pseudocontractive mapping and an averaged mapping involving $k$-strictly pseudocontractive mapping. Numerical examples were presented to illustrate the strong convergence of the algorithms. In both examples shown above, it was observed that Algorithm (3.1) converges faster than Algorithm (3.14). Indeed, Algorithm (3.1) reaches the stopping criteria of $\left\|x_{n+1}-x_{n}\right\| \leq 10^{-9}$ faster than Algorithm (3.14). The results presented in this paper extends 
the recent results by Shehu, Iyiola and Ogbuisi [15] from the class of nonexpansive mappings to the much more general class of strictly pseudocontractive mappings.

\section{REFERENCES}

[1] F.E. Browder, W.V.Petryshyn, Construction of fixed points of nonlinear mappings in Hilbert spaces, J. Math. Anal. Appl. 20 (1967), 197-228.

[2] T.L. Hicks, J.R. Kubicek, On the Mann iterative process in Hilbert spaces, J. Math. Anal. Appl. 59 (1977), 498-504.

[3] S. Maruster, The solution by iteration of nonlinear equations in Hilbert spaces, Proc. Amer. Math. Soc. 63 (1977), 69-73.

[4] W.R. Mann, Mean value methods in iteration, Proc. Amer. Math. Soc. 4 (1953), 506-510.

[5] S. Ishikawa, Fixed point by a new iteration method, Proc. Amer. Math. Soc. 44 (1974), 147-150.

[6] L. Qihou, The convergence theorems of the sequence of Ishikawa iterates for hemicontractive mappings, J. Math. Anal. Appl. 148 (1990), 55-62.

[7] H. Zegeye, N. Shahzad and M. A. Alghamdi, Convergence of Ishikawa's iteration method for pseudocontractive mappings, Nonlinear Analysis 74 (2011) 7304-7311.

[8] T.H. Kim, H.K. Xu, Strong convergence of modified Mann iterations, Nonlinear Anal. 61 (2005), 51-60.

[9] X. Qin, J.C. Yao, Weak convergence of a Mann-like algorithm for nonexpansive and accretive operators, J. Inequal. Appl. 2016 (2016), 232.

[10] K. Nakajo, W. Takahashi, Strong convergence theorems for nonexpansive mappings and nonexpansive semigroups, J. Math. Anal. Appl. 279 (2003), 372-379.

[11] P.E. Mainge, S. Maruster, Convergence in norm of modified Krasnoselkii-Mann iterations for fixed points of demicontractive mappings, Appl. Maths. Comput. 217 (2011), 9864-9874.

[12] X. Qin, Y. Su, Strong convergence theorems for relatively nonexpansive mappings in a Banach space, 67 (2007), 1958-1965.

[13] C. Martinez-Yanes, H.K. Xu, Strong convergence of the CQ method for fixed point iteration processes, Nonlinear Anal. 64 (2006), 2400-2411.

[14] C.E. Chidume, N. Shahzad, Weak convergence theorems for a finite family of strict pseudocontractions, Nonlinear Anal. 72 (2010), 1257-1265.

[15] Y. Shehu, O.S. Iyiola, F.U. Ogbuisi, Iterative method with inertial terms for nonexpansive mappings: applications to compressed sensing, Numer. Algor. 83 (2020), 1321-1347.

[16] M.O. Osilike and A. Udomene, Demiclosedness principle and convergence results for strictly pseudocontractive mappings of Browder-Petryshyn type, J. Math. Anal. Appl. 256 (2001), 431-445.

[17] P.E. Mainge, Strong convergence of projected subgradient methods for nonsmooth and nonstrictly convex minimization, Set-Valued Anal. 16 (2008) 899-912.

[18] P.U. Nwokoro, M.O. Osilike, D.F. Agbebaku, E.E. Chima, A.C. Onah, A new Halpern-type averaging algorithm with inertial and error terms for fixed points of asymptotically nonexpansive maps, J. Math. Comput. Sci. 10 (2020), 1538-1558.

[19] H.H. Bauschke, P.L. Combettes, Convex Analysis and Monotone Operator Theory in Hilbert Spaces, Canadian Mathematical Society (CMS) Books in Mathematics, Springer, 2011. 